\title{
Strategies for Representing Metabolic Pathways within Biochemical Systems Theory: Reversible Pathways*
}

\author{
ALBERT SORRIBAS ${ }^{\dagger}$ AND MICHAEL A. SAVAGEAU \\ Department of Microbiology and Immunology, The University of Michigan, Ann Arbor, \\ Michigan 48109-0620
}

Received 6 July 1988; revised 1 October 1988

\begin{abstract}
The search for systematic methods to deal with the integrated behavior of complex biochemical systems has over the past two decades led to the proposal of several theories of biochemical systems. Among the most promising is biochemical systems theory (BST). Recent comparisons of this theory with several others that have recently been proposed have demonstrated that all are variants of BST and share a common underlying formalism. Hence, the different variants can be precisely related and ranked according to their completeness and operational utility. The original and most fruitful variant within BST is based on a particular representation, called an S-system (for synergistic and saturable systems), that exhibits many advantages not found among alternative representations. Even within the preferred S-system representation there are options, depending on the method of aggregating fluxes, that become especially apparent when one considers reversible pathways. In this paper we focus on the paradigm situation and clearly distinguish the two most common strategies for generating an S-system representation. The first is called the "reversible" strategy because it involves aggregating incoming fluxes separately from outgoing fluxes for each metabolite to define a net flux that can be positive, negative, or zero. The second is the "irreversible" strategy, which involves aggregating forward and reverse fluxes through each reaction to define a net flux that is always positive. This second strategy has been used almost exclusively in all variants of BST. The principal results of detailed analyses are the following: (1) All S-system representations predict the same changes in dependent concentrations for a given change in an independent concentration. (2) The reversible strategy is superior to the irreversible on the basis of several criteria, including accuracy in predicting steady-state flux, accuracy in predicting transient responses, and robustness of representation. (3) Only the reversible strategy yields a representation that is able to capture the characteristic feature of amphibolic pathways, namely, the
\end{abstract}

* Material in this paper was presented at the 6th International Conference on Mathematical Modelling, St. Louis, Missouri, 4-7 August 1987, and the 1st IFAC Symposium on Modelling and Control in Biomedical Systems, Venice, Italy, 6-9 April 1988.

${ }^{\dagger}$ Present address: Departament de Ciencies Mediques Rasiques Biostadistica, Facultat de Medicina, Estudi General de Lleida, Universitat de Barcelona, 25007-LLEIDA, Spain. 
reversal of nets flux under physiological conditions. Finally, the results document the wide range of variation over which the S-system representation can accurately predict the behavior of intact biochemical systems and confirm similar results of earlier studies [Voit and Savageau, Biochemistry 26: 6869-6880 (1987)].

\section{INTRODUCTION}

Biochemical pathways are complex dynamic systems that are characterized by a large number of interactions and nonlinear processes. Analysis of these systems requires appropriate mathematical representations to provide a meaningful methodological framework for dealing with the complexity.

The development of such representations has been a major objective of several groups for the past two decades. The result of this effort, until very recently, has been considerable confusion that can be traced to the lack of careful comparisons among the different theories that have been proposed and to the introduction of diverse notation that is often at variance with well-accepted notation in related areas. ${ }^{1}$ However, careful comparisons of these various theories show that they are all variants of a single theory, called biochemical systems theory (BST). This is true by virtue of their sharing a fundamental property - the use, explicitly or implicitly, of the power-law formalism for representing the different processes of the system $[35,36,38,39,41]$.

This formalism was developed in the late 1960s by combining well-known general principles of network theory with the specific characteristics of biochemical processes. The concepts, theory, and methodology that were produced [20-25] provide a very general framework for analysis that includes a structured set of rules for building mathematical representations of complex biochemical systems. The preferred representation in BST is called an S-system (for synergistic and saturable system) and is obtained by first aggregating the several processes affecting a dependent metabolite into two processes, one accounting for its synthesis and another for its degradation. Following this procedure, the rate of each of the aggregate processes is described by a product of power-law functions, which produces the S-system representation. Once the mathematical representation has been derived, the various parameters of the S-system are evaluated at a nominal operating point to produce an exact characterization of the system at that point.

The S-system is one of several possibilities for using the power-law formalism to represent biochemical systems. Its principal advantages lie in

\footnotetext{
${ }^{1}$ In particular, metabolic control theory [6-8, 12-15, 43] and flux-oriented theory [2-4] have not been critically tested, nor have they been distinguished from previously existing theory, e.g., by comparison of their predictions with those of earlier approaches to the same problem. (See [32], [37], [40], [42] for references and brief discussion of these issues, and [34]-[36], [38], [39] for detailed analysis and comparisons.)
} 
its ability to provide an explicit steady-state solution in symbolic form and to predict accurately the behavior of the system over a wide range of variation about the operating point at which the S-system representation is built $[21,28,35,36,38,39,41]$. However, even within this form of representation there are alternative strategies for aggregation that produce alternative S-system representations for the same pathway. Although these alternatives are equivalent at the operating point and provide the same characterization of the system at this point, they differ in their ability to predict the behavior of the system in the vicinity of such a point. These differences become particularly apparent in dealing with reversible processes.

Although all processes are in principle reversible, there are many reactions that for all practical purposes can be considered irreversible. Living systems typically operate far from thermodynamic equilibrium. If a cell has need to synthesize a given substance under one set of conditions and to utilize or degrade that substance under another set of conditions, it will generally do so via two entirely separate pathways that operate in an essentially unidirectional fashion. Pathways of the first type are known as biosynthetic or anabolic; those of the second type are known as catabolic. For example, in certain strains of Salmonella typhimurium there is a histidine catabolic pathway $[16,17]$ that is entirely separate from the histidine biosynthetic pathway [1]. This pattern is so widely observed in cellular physiology that it has been elevated to the status of an "organizational principle." Nevertheless, there are important pathways in the cell that do function in a reversible fashion and thus are organized according to different principles; these pathways are called amphibolic. The most notable example is the glycolytic pathway in which significant portions are also used for gluconeogenesis by some types of cells [5, 19].

In this paper we compare the two principal strategies for S-system representation of reversible pathways. The "reversible" strategy involves aggregation of incoming fluxes separately from outgoing fluxes for each metabolite to define a net flux that can be positive, negative, or zero; this amounts to choosing the set of fluxes with the same sense toward a given metabolic as the building block for construction of the S-system representation. The "irreversible" strategy involves aggregation of forward and reverse fluxes through each reaction to define a net flux that is always positive; this amounts to choosing the net flux through a given reaction as a building block.

The criteria for these comparisons are four: (1) accuracy in predicting values of the concentration variables at steady states around the nominal operating point, (2) accuracy in predicting values of the flux variables at steady states around the nominal operating point, (3) accuracy in predicting transient responses between two such steady states, and (4) robustness of the representation. 
The results of detailed and rigorous comparisons show that all possible S-system representations of a biochemical pathway predict the same values for the concentration variables at steady states around the nominal operating point. On the other hand, the two principal strategies yield very different predictions for steady-state fluxes and transient responses. The reversible strategy is always more accurate and in particular is the only strategy capable of representing thermodynamic equilibrium. These results confirm and extend early results on the optimum procedure for aggregation [41]. Other limitations associated with alternative aggregation procedures are documented elsewhere $[38,39]$. Finally, our results show that the more accurate representation is also the more robust. Near equilibrium, parameter sensitivities using the reversible strategy are on average about an order of magnitude smaller than those using the irreversible strategy, which means that slight changes in the system produce only slight changes in its S-system representation when the reversible strategy is used. Thesc results also make clear that the parameter sensitivities are not simply a property of the biochemical system but reflect the mathematical representation as well. Hence, high parameter sensitivities associated with a given process may not be indicative of a "control" point in the biochemical system, but only of an inappropriate mathematical representation for the system.

\section{METHODOLOGY}

Analysis of complex biochemical systems can be broken down into three distinct steps: mathematical representation of the system, steady-state analysis, and dynamic analysis. There is a well-developed methodology for each of these steps in biochemical systems theory.

\subsection{S-SYSTEM REPRESENTATION}

The S-system representation is built by first aggregating kinetic descriptions for the different processes affecting a metabolite $X_{i}$; those that account for the synthesis of $X_{i}$ yield a net rate law $V_{i}$, and those that account for its degradation yield a net rate law $V_{-i}$. Each of these net rate laws is then represented by a product of power-law functions, which produces the S-system equations ${ }^{2}$

$$
\frac{d X_{i}}{d t}=V_{i}-V_{-i}=\alpha_{i} \prod_{j=1}^{n+m} X_{j}^{g_{i j}}-\beta_{i} \prod_{j=1}^{n+m} X_{j}^{h_{i j}}, \quad i=1, \ldots, n,
$$

\footnotetext{
${ }^{2}$ We shall omit for simplicity discussion of situations involving constraints or aggregate concentration variables. In any case, these additional features can be handled in a straightforward manner that leaves the form of the resulting equations unchanged. For details, see Savageau [28, 29], Savageau et al. [35, 36], and Sorribas and Savageau [38, 39].
} 
where $n$ is the number of dependent concentration variables in the system and $m$ is the number of independent concentration variables.

The parameters of the power-law formalism are defined as follows:

$$
\begin{aligned}
g_{i j} & =\left(\frac{\partial V_{i}}{\partial X_{j}}\right)_{0}\left(\frac{X_{j 0}}{V_{i 0}}\right), & h_{i j} & =\left(\frac{\partial V_{-i}}{\partial X_{j}}\right)_{0}\left(\frac{X_{j 0}}{V_{-i 0}}\right), \\
\alpha_{i} & =V_{i 0} \prod_{j=1}^{n+m} X_{j 0}^{-g_{i j}}, & \beta_{i} & =V_{-i 0} \prod_{j=1}^{n+m} X_{j 0}^{-h_{i j}} .
\end{aligned}
$$

They result from Taylor series expansions of $V_{i}$ and $V_{-i}$ in a logarithmic coordinate system at an operating point signified by the subscript $0[21,25$, 28]. The parameters are readily identified as the rate constant $\left(\alpha_{i}\right.$ and $\left.\beta_{i}\right)$ and the kinetic orders $\left(g_{i j}\right.$ and $\left.h_{i j}\right)$ associated with each of the aggregate rate laws (synthesis and degradation) in the system. The numerical values of the parameters reflect the underlying mechanisms and the choice of operating point at which the system is characterized exactly.

The accuracy with which the S-system representation predicts the behavior of biochemical systems for variations about the nominal operating point depends upon the particular characteristics of the system. However, available evidence suggests that $\mathrm{S}$-systems provide an accurate representation over a wide range of variations, comparable to those seen for biochemical variables in situ $[28,38,39,41]$.

\subsection{STEADY-STATE SOLUTIONS}

A major advantage of the S-system representation resides in its ability to provide an explicit steady-state solution in symbolic form [21]. From Eq. (1), the steady-state description of the system can be written in logarithmic coordinates:

$$
[A] y]=b]
$$

where $y$ ] is a vector of dimension $n+m$ with elements given by $y_{i}=\log X_{i}$, $[A]$ is a matrix of dimension $n \times(n+m)$ with elements $a_{i j}=g_{i j}-h_{i j}$ $(i=1, \ldots n ; j=1, \ldots, n+m)$, and $b]$ is a vector of dimension $n$ with elements given by $b_{i}=\log \left(\beta_{i} / \alpha_{i}\right)$.

To obtain a unique nonzero steady state, it is necessary to separate dependent and independent concentration variables. Rearranging Eq. (3) yields

$$
\left.\left.\left.[A]_{d} y\right]_{d}+[A]_{i} y\right]_{i}=b\right],
$$

where the subscripts $d$ and $i$ signify that the corresponding matrices (vectors) contain only kinetic orders (logarithms of concentrations) for dependent and independent concentrations, respectively. One can solve explicitly 
for the $n$ dependent variables in terms of the parameters and the $m$ independent variables, provided the matrix $[A]_{d}$ is nonsingular. The resulting steady-state solution for Eq. (1) then can be written [24]

$$
\left.\left.\left.y]_{d}=-[A]_{d}^{-1}[A]_{i} y\right]_{i}+[A]_{d}^{-1}[b]=[L]_{y}\right]_{i}+[M] b\right] .
$$

The matrices $[M]$ and $[L]$, whose elements consist of various combinations of all the kinetic orders and only the kinetic orders of the system, contain information regarding changes in dependent concentrations in response to changes in independent concentrations (logarithmic gains), rate constants (rate-constant sensitivities), and kinetic orders (kinetic-order sensitivities). These logarithmic gains and parameter sensitivities allow a complete characterization of the system's steady-state behavior and are the basic components of the methodology for analyzing metabolic pathways by means of an S-system representation [25, 28].

This approach has been used successfully in the analysis of many different types of biochemical systems, including feedback and feedforward mechanisms of control in biosynthetic pathways [25-28, 33], circuits for inducible gene expression $[28,30,31]$, network regulation of the immune response $[9,10]$, and enzyme-enzyme interactions $[38,39]$.

\subsection{DYNAMIC SOLUTIONS}

The dynamic behavior of a biochemical system is determined by a set of nonlinear differential equations that generally is difficult to solve. However, the systematic structure provided by the S-system representation is a major advantage when it comes to obtaining dynamic solutions. In this representation, a mathematical model for any biochemical system always has the same form, that of Eq. (1). For each specific case, only the numbers of independent and dependent variables and the numerical values of the parameters change. This consistency of form allows one to develop very efficient computer algorithms for obtaining the dynamic solutions as well as the steady-state solutions referred to earlier.

Such algorithms have been implemented in a comprehensive program called ESSYNS (for Evaluation and Simulation of SYNergistic Systems), which runs on an IBM PC/AT machine [Voit et al., in preparation]. This program provides dynamic solutions one to two orders of magnitude faster than conventional algorithms under the same conditions of local tolerance and global accuracy [11; Irvine and Savageau, in preparation]. We have used ESSYNS throughout the studies reported in this paper.

\section{THEORY}

We shall first deduce the number of possible S-system representations. Then we shall show that all of these yield the same steady-state solutions for 
the dependent concentrations. Finally we shall focus more specifically on a pathway of reversible reactions and present the two principal strategies for S-system representation.

\subsection{NUMBER OF POSSIBLE S-SYSTEM REPRESENTATIONS}

Let us consider an arbitrary biochemical system with $n$ dependent concentrations, $m$ independent concentrations, and at most $p$ parallel reactions unidirectionally converting any one metabolite into any other. The mass balance equations for the dependent metabolites $X_{i}$ can then be written as the difference between two summations, the first consisting of $r_{i}$ terms and the second of $s_{i}$ terms:

$$
\frac{d X_{i}}{d t}=\sum_{j=1}^{n+m} \sum_{k=1}^{p} v_{j i k}-\sum_{j=1}^{n+m} \sum_{k=1}^{p} v_{i j k}, \quad i=1, \ldots, n,
$$

where $v_{i j k}$ is the unidirectional rate of utilization of $X_{i}$ for the production of $X_{j}$ via the $k$ th parallel reaction. (For simplicity, and without loss of generality, we consider the stoichiometric values to be unity. If one wishes to consider values different from unity, one simply multiplies the rates in the first summation by the appropriate stoichiometric factors [21].)

As discussed in Section 2, one builds an S-system representation of a particular biochemical system by aggregating individual rate laws to form two net rate laws $V_{i}$ and $V_{-i}$ for each dependent concentration. This can be done in a number of ways. For example, one might aggregate three terms from the first sum with two terms from the second and then define this new sum as $V_{i}$. The only restrictions are that (1) each term of Eq. (5) must be assigned to either $V_{i}$ or $V_{-i}$, but not both; (2) at least one term must be assigned to each $V_{i}$ and $V_{i}$; and (3) the resulting $V_{i}$ and $V_{i}$ must be positive quantities at the operating point.

The number of different combinations of terms that meet the above restrictions can be calculated as follows. If $V_{i}$ were to contain all the terms in all combinations, there would be $2^{r_{i}+s_{i}}$ combinations. (For any given combination of terms assigned to $V_{i}, V_{-i}$ will automatically contain the complement of the full set.) However, $V_{i}$ cannot contain all the terms, nor can it contain none of the terms. The above number must therefore be reduced by 2 . Furthermore, by symmetry, half of the combinations will result in $V_{1}$ and $V_{-t}$, values that are negative at the operating point, so this number must be reduced further by a factor of 2 to

$$
2^{r_{i}+s_{i}-1}-1
$$

Finally, since there are $n$ equations for the dependent concentrations, the 
total number of possible S-system representations is

$$
N=\prod_{i=1}^{n}\left(2^{r_{i}+s_{i}-1}-1\right) \text {. }
$$

Although all of these will have the same S-system form, the parameter values will differ depending upon the particular type of aggregation used. These differences in parameter values will in general lead to different predictions for the behavior of the biochemical system and hence to the question of which representation is most accurate. However, before addressing these questions for pathways of reversible reactions, we will first show that all possible S-system representations predict the same same-state behavior for the dependent concentrations of the system.

\subsection{IDENTICAL STEADY-STATE SOLUTIONS FOR DEPENDENT CONCENTRATIONS}

Although one might expect steady-state solutions for dependent concentrations to differ with the choice of S-system representation, this is not the case, as can be seen most readily by considering one of the steady-state equations represented in $\mathrm{Eq}$. (4). At the operating point all representations are equivalent and

$$
y_{i 0}=\sum_{k=n+1}^{n+m} L_{i k} y_{k 0}+\sum_{j=1}^{n} M_{i j} b_{j}, \quad i=1, \ldots, n
$$

The values of $y_{i 0}$ and $y_{k 0}$ are fixed independent of the representation. Similarly, the elements $L_{i k}$, which are the logarithmic gains expressing percentage change in dependent concentrations in response to a $1 \%$ change in an independent concentration, and which are given by

$$
L_{i k}=\left(\frac{\partial y_{i}}{\partial y_{k}}\right)_{0}=\left(\frac{\partial X_{i}}{\partial X_{k}}\right)_{0}\left(\frac{X_{k 0}}{X_{i 0}}\right)
$$

have fixed values independent of the representation. Hence, the second term on the right-hand side of Eq. (7) also must have a fixed value independent of the representation, even though the values of the individual $M_{i j}$ elements and the individual $b_{j}$ elements will differ with the aggregation procedure used.

At steady states other than the operating point,

$$
y_{i}=\sum_{k=n+1}^{n+m} L_{i k} y_{k}+\sum_{j=1}^{n} M_{i j} b_{j}, \quad i=1, \ldots, n,
$$


and inserting the fixed value for the second term, determined from Eq. (7), yields

$$
y_{i}=y_{i 0}+\sum_{k=n+1}^{n+m} L_{i k}\left(y_{k}-y_{k 0}\right), \quad i=1, \ldots, n
$$

The value of the independent variable $y_{k}$ also is fixed independent of the representation. Hence, we have the following general result ${ }^{3}$ :

All possible S-system representations of a given biochemical system exhibit the same steady-state behavior for the dependent concentrations.

The accuracy in predicting values of the concentration variables at steady states around the nominal operating point will be identical for all possible $\mathrm{S}$-system representations. However, because the net rate laws will differ, depending upon the aggregation procedure used and the specifics of the biochemical system under study, one can expect various $\mathrm{S}$-system representations to reflect these differences when compared on the basis of the other three criteria: accuracy in predicting values of the flux variables at steady states around the nominal operating point, accuracy in predicting transient responses between two such steady states, and robustness of representation. In order to examine these issues in detail, we shall now focus on a pathway of reversible reactions.

\subsection{ALTERNATIVE S-SYSTEM REPRESENTATIONS FOR REVERSIBLE PATHWAYS}

Amphibolic pathways, which exhibit a reversal of net flux through the system, consist of reactions that may be considered either as single bidirectional processes or as a combination of two unidirectional processes of opposite sense (see Figure 1). Displacement of the ratio of substrate and product concentrations, which are considered independent variables, from its value at thermodynamic equilibrium provides the driving force for generating flux in one direction or the other. The corresponding mass balance equations are

$$
\frac{d X_{i}}{d t}=v_{i-1, i}+v_{i+1, i}-v_{i, i-1}-v_{i, i+1}, \quad i=1, \ldots, n
$$

where $i-1=n+2$ if $i=1$ and each of the unidirectional rate laws may be a

\footnotetext{
${ }^{3}$ It should be noted that some forms of aggregation will fail to produce a valid S-system representation at isolated operating points; namely, those that yield a $V_{i}$ or $V_{-i}$ equal to zero. If one changes the operating point slightly, then it becomes possible to generate a valid S-system representation. We shall see examples of this in Sections 3.3 and 4.
} 


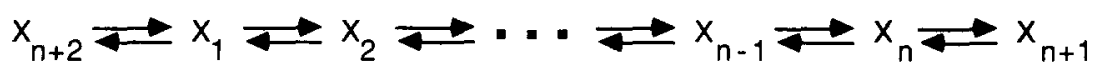

FIG. 1. Amphibolic pathway with metabolites $X_{i}(i=1, \ldots, n+2)$. The concentrations of product, $X_{n+1}$, and of substrate, $X_{n+2}$, are independent variables held constant by the environment. The numbering of concentration variables in this figure is done by convention so that the $n$ dependent variables range from 1 to $n$, and the $m$ independent variables range from $n+1$ to $n+m$.

positive but complex nonlinear function of the concentration variables in the pathway.

In this case, since $r_{i}=s_{i}=2$, there are seven possibilities for aggregating the individual rate laws associated with a given metabolite $X_{i}$. However, of these seven possibilities there are only two that are commonly used in representing reversible pathways. In one, the reversible strategy, the set of fluxes with the same sense toward an individual metabolite is considered a building block in the description, whereas in the other, the irreversible strategy, the net flux through an individual reaction is considered a building block.

The Reversible Strategy. Aggregation according to the reversible strategy (R), i.e., aggregating the sets of fluxes with the same sense toward the metabolite $X_{i}$, which is the same as aggregating unidirectional rate laws of the same sign, yields

$$
\frac{d X_{i}}{d t}=\left(v_{i-1, i}+v_{i+1, i}\right)-\left(v_{i, i-1}+v_{i, i+1}\right), \quad i=1, \cdots, n
$$

from which it can be seen that

$$
V_{i}=\left(v_{i-1, i}+v_{i+1, i}\right) \quad \text { and } \quad V_{-i}=\left(v_{i, i-1}+v_{i, i+1}\right), \quad i=1, \ldots, n
$$

Each of these aggregate rate laws then can be replaced by the appropriate product of power-law functions to yield an S-system representation of Eq. (9).

$$
\frac{d X_{i}}{d t}=\alpha_{i}^{\mathrm{R}} X_{i-1}^{g_{i, j}^{\mathrm{R}}} X_{i}^{g_{i i}^{\mathrm{R}}} X_{i+1}^{g_{i+1}^{\mathrm{R}}}-\beta_{i}^{\mathrm{R}} X_{i-1}^{h_{i, 1}^{\mathrm{R}}} X_{i}^{h_{i i}^{\mathrm{R}}} X_{i+1}^{h_{i+1+1}^{\mathrm{R}}}, \quad i=1, \ldots, n .
$$

The solution of these equations [Eq. (4)] gives the solution for the flux variables by a straightforward secondary calculation. The net flux through the pathway in steady state can be determined at any step; we shall simply 
indicate the determination at the end of the pathway.

$$
\begin{aligned}
V_{\mathrm{net}}^{\mathrm{R}} & =V_{n+1}^{\mathrm{R}}-V_{-(n+1)}^{\mathrm{R}} \\
& =\alpha_{n+1}^{\mathrm{R}} X_{n}^{g_{n+1, n}^{\mathrm{R}}} X_{n+1}^{g_{n+1, n+1}^{\mathrm{R}}}-\beta_{n+1}^{\mathrm{R}} X_{n+1, n}^{h_{n+1}^{\mathrm{R}}} X_{n+1}^{h_{n+1, n+1} \mathrm{R}} .
\end{aligned}
$$

In this strategy, the net flux is represented by the difference between two products of power-law functions and, although each of these is always positive, the difference allows for a reversal in the direction of net flux,

The Irreversible Strategy. Aggregation of the individual rate laws in Eq. (9) according to the irreversible strategy (I), i.e., aggregating the forward and reverse fluxes through each reaction to produce a net flux, yields

$$
\frac{d X_{i}}{d t}=\left(v_{i-1, i}-v_{i, i-1}\right)-\left(v_{i, i+1}-v_{i+1, i}\right), \quad i=1, \ldots, n
$$

The corresponding aggregate rate laws then can be identified as

$$
V_{i}=\left(v_{i-1, i}-v_{i, i-1}\right) \quad \text { and } \quad V_{-i}=\left(v_{i, i+1}-v_{i+1, i}\right), \quad i=1, \ldots, n \text {. }
$$

Each of these aggregate rate laws is replaced by the appropriate product of power-law functions to produce an S-system representation of Eq. (9).

$$
\frac{d X_{i}}{d t}=\alpha_{i}^{\mathrm{I}} X_{i-1}^{g_{i, j}^{\mathrm{I}}-1} X_{i}^{\mathrm{g}_{i i}^{\mathrm{I}}}-\beta_{i}^{\mathrm{I}} X_{i}^{h_{i i}^{\mathrm{I}}} X_{i+1}^{h_{i}^{\mathrm{I}}}, \quad i=1, \ldots, n .
$$

Again, the net flux through the pathway in steady state can be determined at any step, but we shall indicate only the determination at the end of the pathway.

$$
V_{\text {net }}^{\mathrm{I}}=V_{-n}^{\mathrm{I}}=\beta_{n}^{\mathrm{I}} X_{n}^{h_{n n}^{\mathrm{I}}} X_{n+\mathrm{I}^{+1}}^{h_{n}^{\mathrm{I}}}
$$

When one takes into account that all concentration variables are positive, it becomes clear that the net flux in Eq. (15) can have only positive values. This represents an important limitation of the irreversible strategy because the resulting description of the system is unable to account for the reversal in direction of net flux that occurs in amphibolic pathways. This limitation is not found with the alternative reversible strategy.

Although the description according to the reversible strategy [Eqs. (11) and (12)] appears to involve more parameters than that according to the irreversible strategy [Eqs. (14) and (15)], not all parameters in the reversible strategy are independent. In fact, one can show that the number of independent parameters is the same in each case. This has been seen previously in 
other comparisons of alternative strategies for representation within the power-law formalism [41].

With thermodynamic equilibrium as an operating point there is no $\mathrm{S}$-system representation according to the irreversible strategy. The aggregate rate laws $V_{i}^{\mathrm{I}}$ and $V_{-i}^{\mathrm{I}}$ must have value zero at equilibrium, which makes it impossible to represent them as power laws since computation of the parameter values involves division by zero [see Eq. (2)]. The reversible strategy avoids these problems and provides an appropriate S-system representation at all operating points, including thermodynamic equilibrium.

If one can avoid thermodynamic equilibrium, then following these two strategies leads to two alternative S-system representations of the same metabolic pathway. These representations are equivalent at the operating point. Moreover, as we have shown in Section 3.2, predictions of dependent concentrations at steady states other than the operating point will be the same regardless of the strategy used.

However, the accuracy with which the alternative representations are able to predict the response of the system to changes in its environment (variation in values of the independent variables), and the robustness with which the alternative representations respond to changes in their structure (variation in values of the parameters), will depend on how well the alternative aggregation procedures capture the main characteristics of the individual processes. In particular, accuracy depends on the sensitivity of the steady-state solution with respect to changes in the parameters of the S-system representation, which in turn is a function of both the operating point and the kinetic properties of the underlying mechanisms [18, 38, 39].

In order to compare the alternative strategies rigorously, and in detail, we turn to the analysis of a particular system in which specific mechanisms are assumed for each of the individual reactions.

\section{RESULTS}

\subsection{DEFINITION OF A SPECIFIC AMPHIBOLIC PATHWAY}

The mass balance equations for the specific system shown in Figure 2 are

$$
\frac{d X_{1}}{d t}=v_{41}-v_{14}-v_{12}+v_{21}, \quad \frac{d X_{2}}{d t}=v_{12}-v_{21}-v_{23}+v_{32}
$$

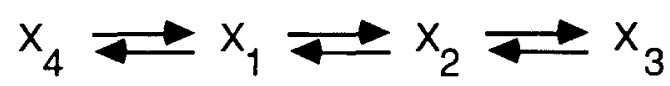

Fig. 2. Amphibolic pathway with $n=2$. The independent concentrations $X_{3}$ and $X_{4}$ are held constant by the environment. 
TABLE 1

Kinetic Parameters for the Rate Laws of the Amphibolic Pathway in Figure $2^{\mathrm{a}}$

\begin{tabular}{ccccc}
\hline \multirow{2}{*}{$\begin{array}{c}\text { Reaction } \\
(i)\end{array}$} & \multicolumn{4}{c}{ Kinetic parameter $^{\mathrm{b}}$} \\
\cline { 2 - 5 } & $\boldsymbol{V}_{i}$ & $K_{i}$ & $V_{-i}$ & $K_{-i}$ \\
\hline $\mathbf{1}$ & 60.0 & 1.00 & 480 & 8.00 \\
2 & 50.0 & 1.00 & 300 & 6.00 \\
3 & 70.0 & 0.500 & 700 & 5.00 \\
\hline
\end{tabular}

${ }^{a}$ See Eq. (17).

${ }^{b}$ Units are $\mu \mathrm{M} \mathrm{s}^{-1}$ for the maximal velocities and $\mu \mathrm{M}$ for the Michaelis constants.

For purposes of illustration, the functional form of the individual nonlinear rate laws is assumed to be

$$
\begin{aligned}
& v_{i, i, i}=\frac{V_{i} X_{i-1} / K_{i}}{1+X_{i-1} / K_{i}+X_{i} / K_{-i}} \\
& v_{i, i-1}=\frac{V_{-i} X_{i} / K_{-i}}{1+X_{i-1} / K_{i}+X_{i} / K_{-i}}
\end{aligned}
$$

These equations describe idealized reversible Michaelis-Menten reactions. The values for the parameters in this representation of the pathway, which constitutes our reference system for comparing the alternative S-system representations, are summarized in Table 1 . For convenience, these values have been chosen consistent with a value of $K_{\text {eq }}=1$ for the overall pathway. This choice is not critical; other values lead to the same qualitative results, although they may differ in quantitative detail.

Displacement of this system from thermodynamic equilibrium is measured by the independent variable

$$
\Gamma=K_{\text {eq }} X_{4} / X_{3},
$$

where $K_{\text {eq }}$ is the equilibrium constant between the substrate $X_{4}$ and the product $X_{3}$. The value of $\Gamma$ is unity at equilibrium. A value greater than unity corresponds to flux in the forward (rightward) direction, while a value less than unity corresponds to flux in the reverse (leftward) direction.

The behavior of this Michaelis-Menten system for different values of $\Gamma$ is shown in Figure 3. In each case, the value of the substrate concentration $X_{4}$ has been fixed while the product concentration $X_{3}$ has been varied to change the mass action ratio. The steady-state values have been determined by computer solution of the system equations using ESSYNS. 

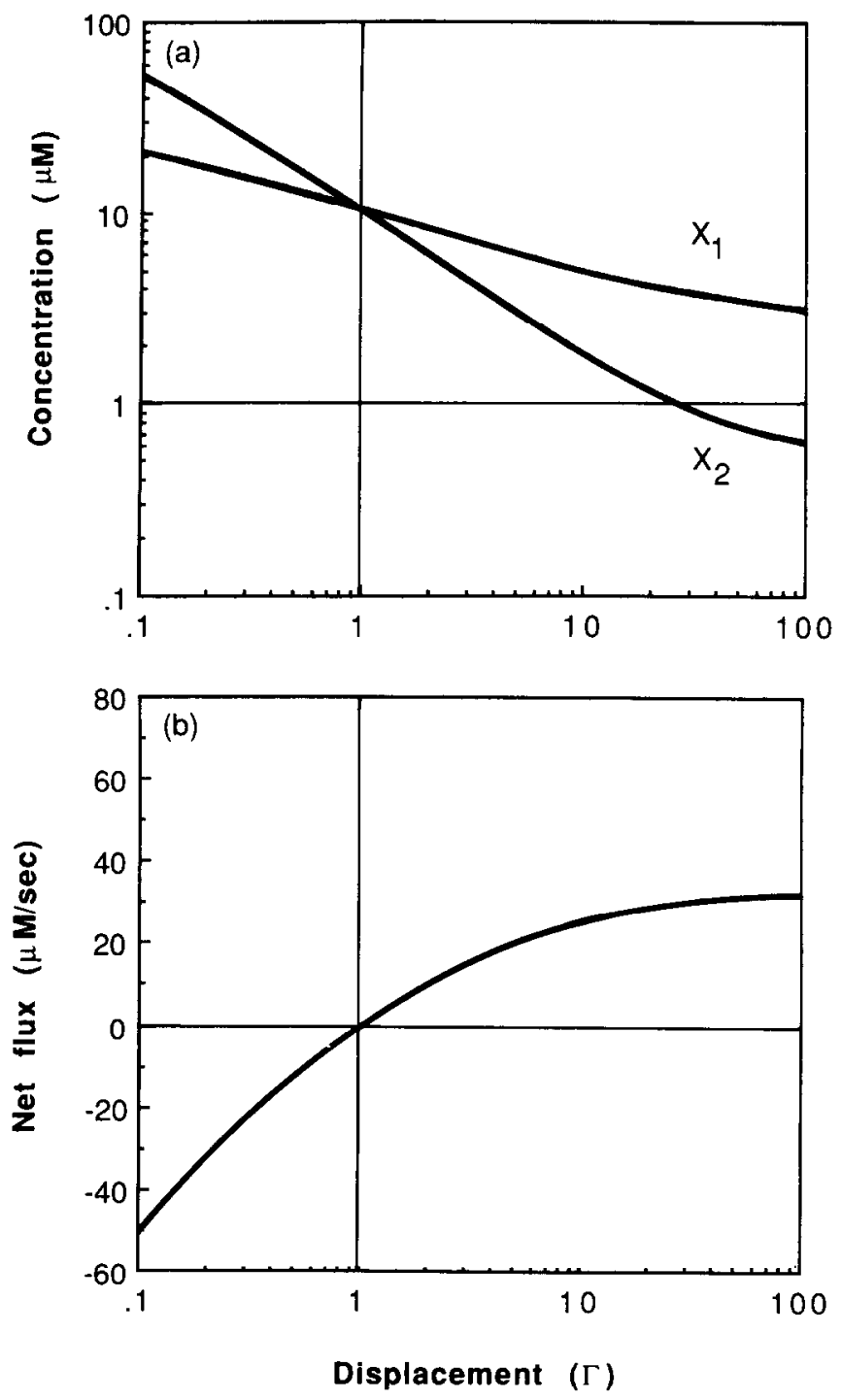

FIG. 3. Steady-state behavior of the amphibolic pathway in Figure 2 as a function of the displacement from equilibrium $\Gamma$. The concentration of substrate, $X_{4}$, is fixed at 10 $\mu \mathrm{M}$, the concentration of product, $X_{3}$, varies from 0.1 to $100 \mu \mathrm{M}$, and $K_{\text {eq }}=1$. (a) Dependent concentrations $X_{1}$ and $X_{2}$. (b) Net flux from $X_{4}$ to $X_{3}$. 


\subsection{ALTERNATIVE S-SYSTEM REPRESENTATIONS OF THE AMPHIBOLIC PATHWAY}

The S-system representations generated by the reversible and irreversible strategies are derived according to the general procedure discussed in Section 3.3. The mathematical representation in the case of the reversible strategy is

$$
\begin{aligned}
& \frac{d X_{1}}{d t}=\alpha_{1}^{\mathrm{R}} X_{1}^{g_{11}^{\mathrm{R}}} X_{2}^{g^{\mathrm{R}}} X_{4}^{g_{14}^{\mathrm{R}}}-\beta_{1}^{\mathrm{R}} X_{1}^{h_{11}^{\mathrm{R}}} X_{2}^{h_{12}^{\mathrm{R}}} X_{4}^{h_{14}^{\mathrm{R}}} \\
& \frac{d X_{2}}{d t}=\alpha_{2}^{\mathrm{R}} X_{1}^{\mathrm{R}{ }^{\mathrm{R}}} X_{2}^{g_{22}^{\mathrm{R}}} X_{3}^{\mathrm{R}_{23}^{\mathrm{R}}}-\beta_{2}^{\mathrm{R}} X_{1}^{h_{21}^{\mathrm{R}}} X_{2}^{h_{22}^{\mathrm{R}}} X_{3}^{h_{23}^{\mathrm{R}}}
\end{aligned}
$$

and in the case of the irreversible strategy it is

$$
\begin{aligned}
& \frac{d X_{1}}{d t}=\alpha_{1}^{\mathrm{I}} X_{1}^{\mathrm{g}_{11}^{\mathrm{I}}} X_{4}^{g_{14}^{\mathrm{I}}}-\beta_{1}^{\mathrm{I}} X_{1}^{h_{11}^{1}} X_{2}^{h_{12}^{\mathrm{I}}} \\
& \frac{d X_{2}}{d t}=\alpha_{2}^{\mathrm{I}} X_{1}^{g_{11}^{\mathrm{I}}} X_{2}^{g_{22}^{\mathrm{I}}}-\beta_{2}^{\mathrm{I}} X_{2}^{h_{22}^{\mathrm{I}}} X_{3}^{h_{23}^{\mathrm{L}}}
\end{aligned}
$$

From the meaning of the parameters in the S-system representation [see Eq. (2)], it is clear that their values will change according to the operating point chosen for analysis of the system. In turn, the accuracy of each S-system representation will change depending on how well the numerical specification of the S-system at a particular operating point captures the essential characteristics of the individual processes of the system. Accordingly, one must analyze the alternative S-system representations at different operating points. The values for the variables at each of the operating points considered are shown in Table 2. In each case, the values of the parameters can be computed analytically [see Eq. (2)] from the rate laws expressed by

TABLE 2

Concentrations and Net Flux for the Amphibolic Pathway in Figure 2 at Different Operating Points

\begin{tabular}{ccccccc}
\hline $\begin{array}{c}\text { Displacement from } \\
\text { equilibrium }\end{array}$ & \multicolumn{2}{c}{$\begin{array}{c}\text { Independent } \\
\text { variables }\end{array}$} & & \multicolumn{3}{c}{$\begin{array}{c}\text { Dependent } \\
\text { variables }\end{array}$} \\
\cline { 5 - 7 }$(\Gamma)$ & $X_{3}$ & $X_{4}$ & & $X_{1}$ & $X_{2}$ & Net flux \\
\hline 1.00 & 10.0 & 10.0 & & 10.0 & 10.0 & 0.00 \\
1.11 & 9.00 & 10.0 & 9.66 & 9.25 & 1.67 \\
2.00 & 5.00 & 10.0 & 8.00 & 6.00 & 10.0 \\
5.00 & 2.00 & 10.0 & 6.10 & 3.07 & 19.9 \\
10.0 & 1.00 & 10.0 & 5.13 & 1.89 & 25.1 \\
\hline
\end{tabular}

${ }^{a}$ Units are $\mu \mathrm{M}$ for the concentrations and $\mu \mathrm{M} \mathrm{s}^{-1}$ for the net flux. 
TABLE 3

Parameter Values for the S-System Generated by the Reversible Strategy at Different Operating Points

\begin{tabular}{cccccc}
\hline \multirow{2}{*}{$\begin{array}{c}\text { Parameter } \\
\text { value }\end{array}$} & 1 & 1.11 & 2 & 5 & 10 \\
\cline { 2 - 6 }$g_{11}$ & -0.409 & -0.401 & -0.352 & -0.274 & -0.219 \\
$g_{12}$ & 0.388 & 0.380 & 0.337 & 0.264 & 0.211 \\
$g_{14}$ & 0.102 & 0.102 & 0.104 & 0.107 & 0.110 \\
$h_{11}$ & 0.591 & 0.586 & 0.558 & 0.521 & 0.500 \\
$h_{12}$ & -0.0587 & -0.0574 & -0.0500 & -0.0378 & -0.0294 \\
$h_{14}$ & -0.452 & -0.447 & -0.417 & -0.372 & -0.343 \\
$g_{21}$ & 0.0828 & 0.0835 & 0.0888 & 0.103 & 0.120 \\
$g_{22}$ & -0.579 & -0.571 & -0.521 & -0.427 & -0.343 \\
$g_{23}$ & 0.554 & 0.548 & 0.516 & 0.455 & 0.397 \\
$h_{21}$ & -0.311 & -0.304 & -0.267 & -0.209 & -0.172 \\
$h_{22}$ & 0.421 & 0.416 & 0.395 & 0.381 & 0.394 \\
$h_{23}$ & -0.0527 & -0.0520 & -0.0476 & -0.0392 & -0.0314 \\
$\alpha_{1}$ & 73.5 & 73.2 & 71.5 & 67.8 & 64.3 \\
$\beta_{1}$ & 73.9 & 73.2 & 71.5 & 68.2 & 65.6 \\
$\alpha_{2}$ & 87.9 & 87.2 & 82.9 & 75.4 & 69.5 \\
$\beta_{2}$ & 87.9 & 87.3 & 83.3 & 75.5 & 69.9 \\
\hline
\end{tabular}

TABLE 4

Parameter Values for the S-System Generated by the Irreversible Strategy at Different Operating Points

\begin{tabular}{|c|c|c|c|c|c|}
\hline \multirow{2}{*}{$\begin{array}{c}\text { Parameter } \\
\text { value }\end{array}$} & \multicolumn{5}{|c|}{ Value of $\Gamma$ at the operating point } \\
\hline & 1 & 1.11 & 2 & 5 & 10 \\
\hline$g_{11}$ & $-{ }^{\mathrm{a}}$ & -28.6 & -4.08 & -1.63 & -1.11 \\
\hline$g_{14}$ & $-^{a}$ & 28.7 & 4.17 & 1.71 & 1.19 \\
\hline$h_{11}$ & $-^{\mathrm{a}}$ & 22.9 & 3.20 & 1.21 & 0.789 \\
\hline$h_{12}$ & $-^{a}$ & -22.9 & -3.10 & -1.08 & -0.634 \\
\hline$g_{21}$ & $-^{a}$ & 22.9 & 3.20 & 1.21 & 0.789 \\
\hline$g_{22}$ & $-{ }^{a}$ & -22.9 & -3.10 & -1.08 & -0.634 \\
\hline$h_{22}$ & $-^{a}$ & 35.6 & 5.14 & 2.05 & 1.36 \\
\hline$h_{23}$ & $-{ }^{a}$ & -35.6 & -5.07 & -1.92 & -1.16 \\
\hline$\alpha_{1}$ & $-^{\mathbf{a}}$ & 0.515 & 3.32 & 7.31 & 9.83 \\
\hline$\beta_{1}$ & ${ }^{\mathbf{a}}$ & 0.518 & 3.33 & 7.47 & 10.4 \\
\hline$\alpha_{2}$ & $-{ }^{a}$ & 0.518 & 3.33 & 7.47 & 10.4 \\
\hline$\beta_{2}$ & $-^{a}$ & 0.559 & 3.49 & 7.52 & 10.5 \\
\hline
\end{tabular}

${ }^{a}$ No S-system representation based on the irreversible strategy is possible with equilibrium as the operating point. 
Eq. (17). The values of the various parameters for each representation are summarized in Tables 3 and 4.

According to the general result presented in Section 3.2, the representations generated by both the reversible and irreversible strategies yield the same steady-state solutions for the dependent concentrations. As can be seen in Figures $4 \mathrm{c}$ and $4 \mathrm{~d}$, changes in concentrations are accurately predicted for a wide range of variation in the independent variables. However, when thermodynamic equilibrium is chosen as the operating point, the irreversible strategy fails to produce a valid S-system representation of the pathway, while the reversible strategy yields a very accurate representation for the steady-state behavior of the pathway (see Figures $4 a, 4 b$ ).
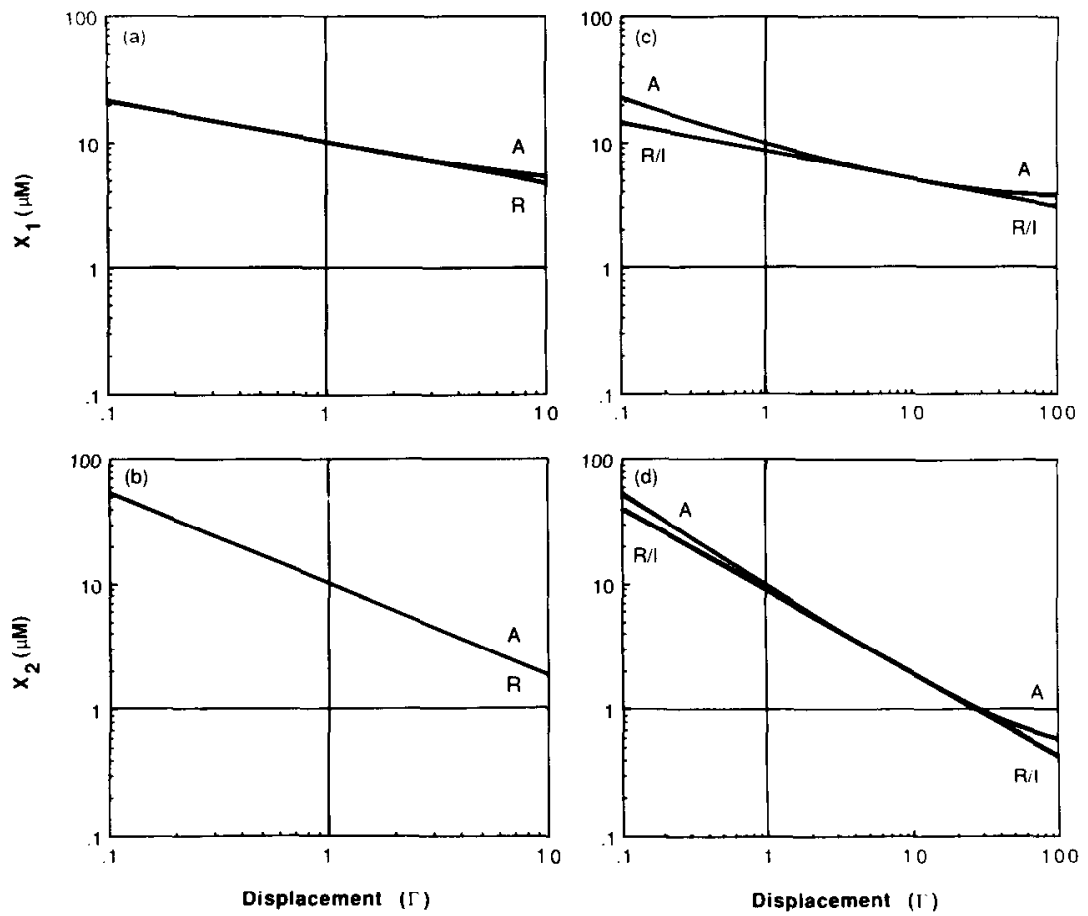

FIG. 4. Accuracy of alternative S-system representations in predicting steady-state concentrations for the amphibolic pathway in Figure 2. Behavior is determined empirically for the amphibolic pathway (A); the same behavior is predicted by $\mathrm{S}$-system representations based on the reversible strategy ( $R$ ) and on the irreversible strategy (I). Comparisons are made with the operating point at equilibrium $\left(\Gamma_{0}=1\right)$ : (a) $X_{1}$ and (b) $X_{2}$. (Note that under these conditions there is no S-system based on the irreversible strategy.) Comparisons are also made with the operating point far from equilibrium $\left(\Gamma_{0}=10\right)$ : (c) $X_{1}$ and (d) $X_{2}$. In all cases, displacement is changed as described in the caption of Figure 3. 

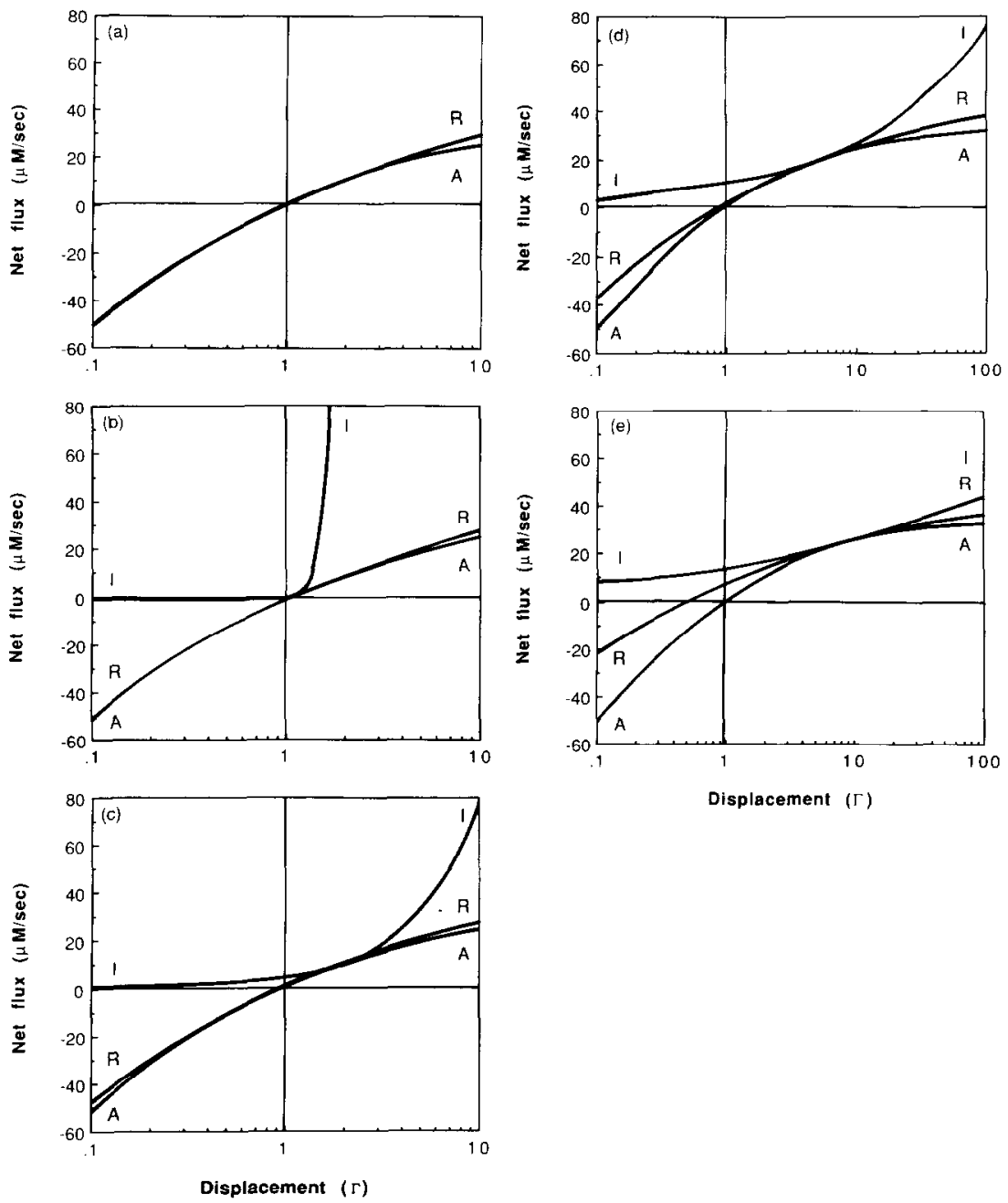

FIG. 5. Accuracy of alternative S-system representations in predicting steady-state flux for the amphibolic pathway in Figure 2. Behavior is determined empirically for the amphibolic pathway (A); the same behavior is predicted by S-system representations based on the reversible strategy ( $R$ ) and on the irreversible strategy (I). Comparisons are made with a series of different operating points: (a) $\Gamma_{0}=1$, (b) $\Gamma_{0}=1.11$, (c) $\Gamma_{0}=2$, (d) $\Gamma_{0}=5$, and (e) $\Gamma_{0}=10$. (Sec Tables 3 and 4 for the corresponding parameter values.) In all cases, displacement is changed as described in the caption of Figure 3. 


\subsection{ACCURACY IN PREDICTING NET FLUX}

Accuracy in predicting steady-state fluxes is the second criterion for comparison given in Section 1. For the net flux through the pathway in Figure 2 this accuracy has been investigated for each of the operating points indicated in Table 2 . In each case, the flux has been predicted over a wide range of values for $\Gamma$, involving at least a tenfold change with respect to the value at the operating point. The results are shown in Figures 5 and 6.

In the first case (Figure 5a), the system is represented at the equilibrium point. According to the previous discussion, the zero value of the net flux at this point makes it impossible to derive a representation according to the irreversible strategy. The reversible strategy, in contrast, yields an S-system

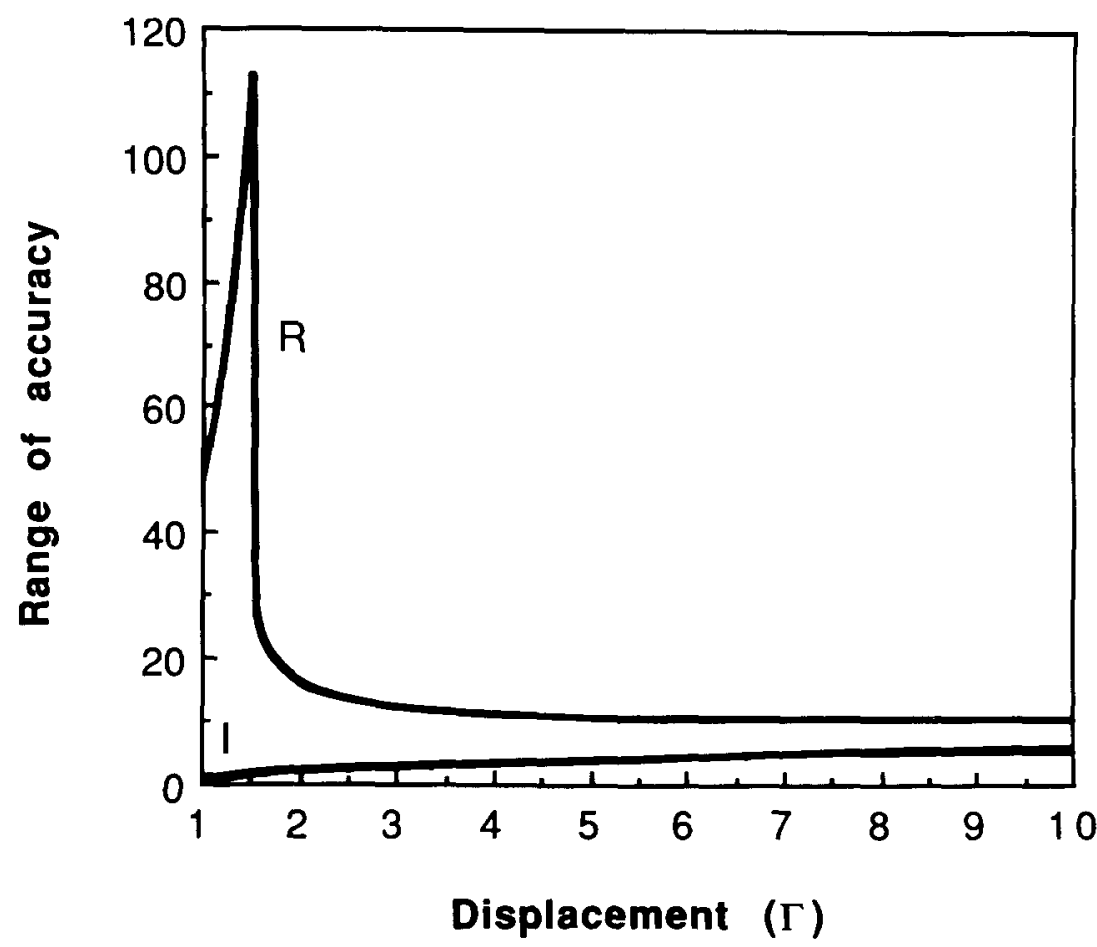

Fig. 6. Range of displacements over which the alternative S-system representations accurately predict the steady-state flux of the amphibolic pathway in Figure 2: $(R)$ S-system representation based on the reversible strategy, (I) S-system representation based on the irreversible strategy. The range is measured by the ratio of the largest to the smallest values of $\Gamma$ about a nominal operating value of $\Gamma_{0}$ that leaves the predicted flux values within $10 \%$ of their empirically determined values. 
representation that predicts the behavior of the system with high accuracy on both sides of the equilibrium point.

When the operating point differs from equilibrium, valid S-system representations become possible with both strategies. As the operating point moves progressively further away from equilibrium, the accuracy of the representation generated by the reversible strategy diminishes (Figures $5 b-e$ ). However, in all cases, this representation is able to predict a change in direction of net flux provided there is an appropriate displacement $\Gamma$. The range over which this representation accurately predicts net flux is very wide when the operating point is near equilibrium but diminishes as the operating point is further and further removed from equilibrium (Figure 6).

In contrast, the representation generated by the irreversible strategy is unable to predict a change in the direction of net flux. This representation always gives a positive value for the net flux regardless of the displacement $\Gamma$; the positive value approaches a minimum of zero as $\Gamma$ tends to zero. For operating points close to equilibrium (e.g., see Figures 5b, 5c), accurate prediction by the representation based on the irreversible strategy is limited to a very narrow range of $\Gamma$ values around the operating point. Although the range of accurate prediction by this representation increases somewhat at operating points further removed from equilibrium (Figures $5 \mathrm{~d}$, $5 \mathrm{e}$ ), it only approaches and never achieves the superior range of the representation based on the reversible strategy (Figure 6).

These results indicate the superiority of the reversible strategy. S-system representations based on this strategy are better able to predict both steadystate values for the net flux of the pathway and the change in direction of net flux associated with equilibrium. These properties are captured even if the operating point is far from equilibrium, although in these cases the accuracy near equilibrium is diminished. However, if the purpose is to represent the change in direction of net flux around equilibrium, then the appropriate operating point for representing such changes will be a point close to the thermodynamic equilibrium of the pathway. Under these conditions the irreversible strategy leads to an inappropriate representation for reversible pathways.

\subsection{ACCURACY IN PREDICTING TRANSIENT RESPONSES BETWEEN STEADY STATES}

The accuracy with which S-system representations of a metabolic pathway predict the transient response between steady states is dependent on the aggregation strategy. Although the different representations yield the same predictions for the steady-state values of the concentrations, each representation captures in a different way the dynamic properties of the individual reactions, and this results in different transient responses between steady states. The transient responses for the system in Figure 2 have been investi- 

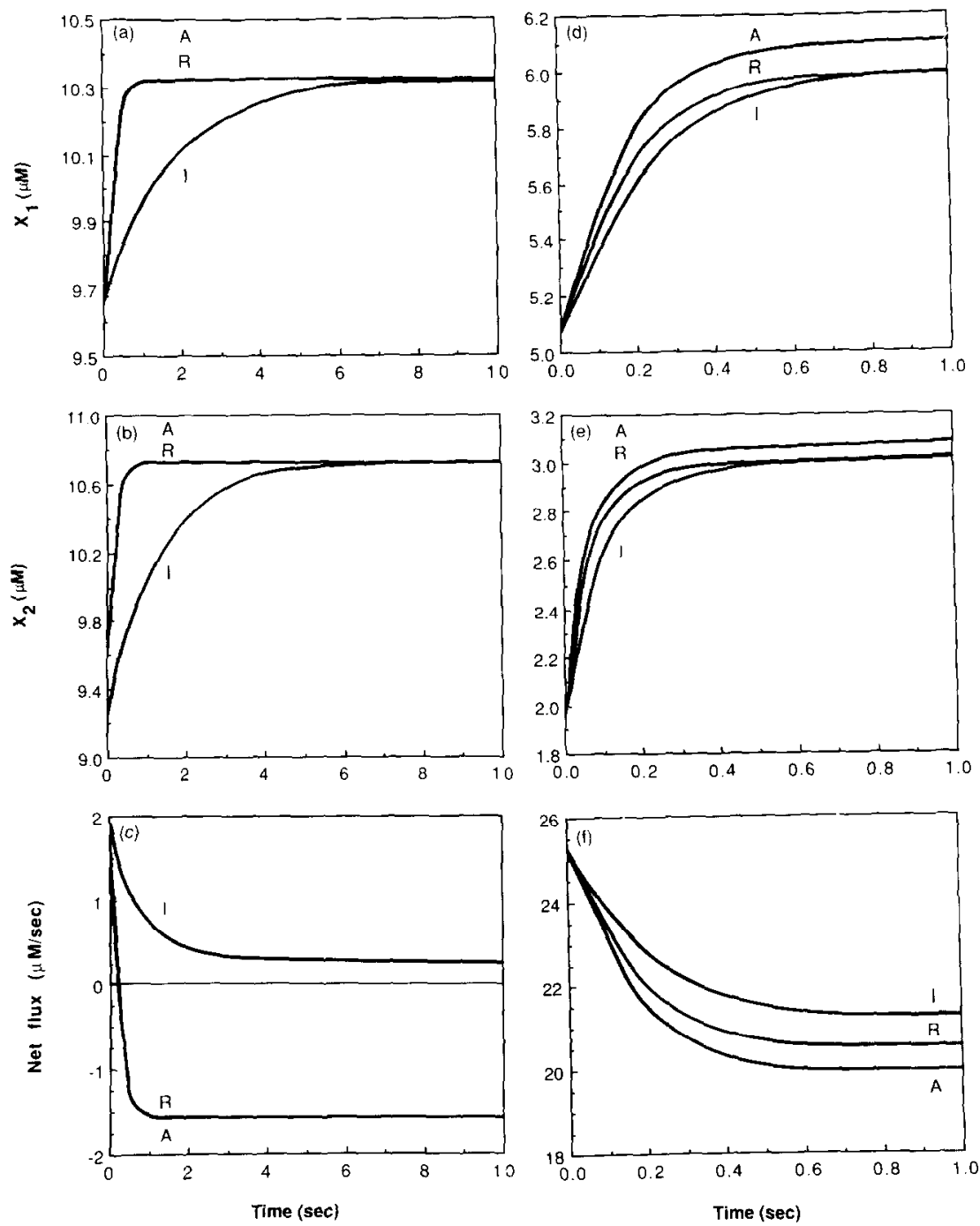

FIG. 7. Accuracy of alternative S-system representations in predicting transient responses for the amphibolic pathway in Figure 2. Behavior is determined empirically for the amphibolic pathway (A); the same behavior is predicted by $\mathrm{S}$-system representations based on the reversible strategy (R) and on the irreversible strategy (I). Before $t=0$ the pathway is at the steady state corresponding to the operating point. At $t=0$ the displacement is perturbed by increasing the concentration of product, $X_{3}$, while the concentration of substrate, $X_{4}$, is held constant at $10 \mu \mathrm{M}$. Comparisons are made with the operating point near equilibrium $\left(\Gamma_{0}=1.11\right.$ and displacement perturbed to $\left.\Gamma=0.91\right)$ : (a) $X_{1}$, (b) $X_{2}$, and (c) net flux into product $X_{3}$. Comparisons also are made with the operating point far from equilibrium ( $\Gamma_{0}=10$ and displacement perturbed to $\Gamma=5$ ): (d) $X_{1}$, (e) $X_{2}$, and (f) net flux into product $X_{3}$. 
gated at two different operating points (Figure 7). In each case, the original steady state is perturbed by increasing the concentration of product, the independent variable $X_{3}$.

The transient response when the operating point is near equilibrium $(\Gamma=1.11)$ leads to a reversal of net flux (Figures $7 \mathrm{a}-\mathrm{c}$ ). In this case, the S-system representation generated by the reversible strategy predicts a transient response nearly identical to that of the amphibolic pathway (errors of $2.3 \%, 1.9 \%$, and $2.5 \%$ in the response times for $X_{1}, X_{2}$, and $V_{\text {net }}$ ), while the irreversible strategy yields a very poor prediction (errors of $447 \%, 850 \%$, and $171 \%$ in the response times for $X_{1}, X_{2}$, and $V_{\text {net }}$ ). Inspection of values for the different parameters in Table 4 shows that this strategy yields a representation with very large values for the kinetic orders when the operating point is close to equilibrium. These anomalous values for the kinetic orders also correlate with high sensitivities, particularly for the flux variables, which have a great influence on the dynamic response of the system. We know from previous results [38] that high parameter sensitivities correlate with low accuracy of representation. Hence, it is not surprising that the irreversible strategy leads to a representation that is unable to accurately predict transient responses in the proximity of equilibrium.

The transient response when the operating point is far from equilibrium $(\Gamma=10)$ is shown in Figures $7 \mathrm{~d}-\mathrm{f}$. In this case, the large displacement from equilibrium at the operating point ensures that the forward component of each reversible reaction will dominate the corresponding reverse component. For higher values of $\Gamma$ the system will be almost equivalent to an irreversible pathway, and therefore both representations will tend to produce the same result. Nevertheless, the $\mathbf{S}$-system representation generated by the reversible strategy (errors of $1.7 \%, 10 \%$, and $6.3 \%$ in the response times for $X_{1}, X_{2}$, and $V_{\text {net }}$ ) is always more accurate than that generated by the irreversible strategy (errors of $37 \%, 74 \%$, and $26 \%$ in the response times for $X_{1}, X_{2}$, and $V_{\text {utet }}$ ), as can be seen from the results presented in Figures $7 \mathrm{~d}-\mathrm{f}$.

These results indicate that the S-system representation generated by the reversible strategy captures the properties of the individual reactions more effectively than that generated by the irreversible strategy, which means more accurate prediction of transient responses between steady states.

\subsection{ROBUSTNESS OF ALTERNATIVE REPRESENTATIONS}

The S-system representation within BST provides a systematic framework for analysis and yields the complete characterization of a system for local variations about an operating point. This means that one can account for the systemic response of all dependent variables to local changes in any of the parameters or any of the independent variables of the system (e.g., see Sorribas and Savageau $[38,39]$ for a detailed treatment of the elements of such an exhaustive characterization). Moreover, within this systematic 
TABLE 5

Complete Characterization of the Amphibolic Pathway in Figure 2 at Equilibrium $(\Gamma=1)$ Using the S-System Representation Generated by the Reversible Strategy

\begin{tabular}{ccccc}
\hline & \multicolumn{4}{c}{ Dependent variables } \\
\cline { 3 - 5 } $\begin{array}{c}\text { Independent } \\
\text { variable }\end{array}$ & $X_{1}$ & \multicolumn{1}{c}{$X_{2}$} & $V_{1}$ \\
$X_{3}$ & \multicolumn{5}{c}{ Logarithmic } \\
$X_{4}$ & 0.328 & 0.735 & 0.151 & 0.155 \\
Parameter & 0.671 & 0.264 & -0.0709 & -0.0972 \\
$g_{11}$ & & & \\
$g_{12}$ & -1.14 & -0.449 & -0.649 & 0.165 \\
$g_{14}$ & 1.08 & 0.425 & 0.614 & 0.156 \\
$h_{11}$ & 0.284 & 0.112 & 0.162 & -0.0411 \\
$h_{12}$ & -1.65 & -0.647 & 0.424 & 0.238 \\
$h_{14}$ & 0.164 & 0.0643 & -0.0421 & -0.0237 \\
$g_{21}$ & 1.26 & 0.495 & -0.325 & -0.182 \\
$g_{22}$ & 0.103 & 0.231 & 0.0474 & 0.0653 \\
$g_{23}$ & -0.721 & -1.61 & -0.331 & -0.456 \\
$h_{21}$ & 0.690 & 1.55 & 0.317 & 0.437 \\
$h_{22}$ & 0.386 & 0.865 & 0.177 & -0.469 \\
$h_{23}$ & -0.524 & -1.17 & -0.241 & 0.637 \\
$\alpha_{1}$ & 0.0657 & 0.147 & 0.0302 & -0.0798 \\
$\beta_{1}$ & 1.21 & 0.476 & 0.688 & -0.175 \\
$\alpha_{2}$ & -1.21 & -0.476 & 0.312 & 0.175 \\
$\beta_{2}$ & 0.541 & 1.21 & 0.249 & 0.343 \\
& -0.541 & -1.21 & -0.249 & 0.657 \\
\hline
\end{tabular}

${ }^{\text {a }}$ The logarithmic gains of $V_{-i}$ are equal to those of $V_{i}$.

${ }^{\mathrm{b}}$ The sensitivities of $V_{-i}$ are equal to those of $V_{i}$,

framework, can one examine the necessary conditions for existence and stability of the steady state in relation to the values of the underlying parameters (e.g., see Savageau et al. [35]).

The complete characterization of the amphibolic pathway in Figure 2 at equilibrium $(\Gamma=1)$ is shown in Table 5 for the S-system representation generated by the reversible strategy. (As we already have shown, there is no such characterization when the irreversible strategy is employed.) The various logarithmic gains and parameter sensitivities are obtained according to the standard procedures described elsewhere (for a review, see Sorribas and Savageau [38]). These results agree with the empirically determined behavior of the amphibolic pathway and illustrate general properties of the S-system representation based on the reversible strategy. 
The relative values of the parameter sensitivities allow one to identify those parameters that must be scrutinized most carefully in experimental studies. As can be seen in Table 5, the relatively high sensitivity of the concentrations to the parameters $g_{11}, g_{12}, g_{22}, g_{23}, h_{11}, h_{14}$, and $h_{22}$ indicates that error in the experimental determination of these parameters will influence the accuracy of the representation to a greater extent than comparable error in the determination of other parameters in the system.

In situ, changes in one parameter will propagate through the system (with gain or attenuation determined by the kinetic orders [24]) and in principle will influence all the dependent variables. Such changes will in turn have an influence on the values of the other parameters in the system. Hence, one can conclude that a representation in which the parameter sensitivities are

\section{TABLE 6}

Complete Characterization of the Amphibolic Pathway in Figure 2

Near Equilibrium $(\Gamma=1.11)$ Using the S-System Representation Generated by the Reversible Strategy

\begin{tabular}{ccccc}
\hline & \multicolumn{4}{c}{ Dependent variables } \\
\cline { 2 - 5 } & $X_{1}$ & $X_{2}$ & $V_{1}$ & $V_{2}$ \\
\hline $\begin{array}{c}\text { Independent } \\
\text { variable }\end{array}$ & \multicolumn{4}{c}{ Logarithmic gains ${ }^{\mathrm{a}}$} \\
$X_{3}$ & 0.327 & 0.736 & 0.149 & 0.155 \\
$X_{4}$ & 0.674 & 0.264 & -0.0672 & -0.0947 \\
Parameter & \multicolumn{4}{c}{ Parameter sensitivities } \\
$g_{11}$ & -1.11 & -0.437 & -0.628 & \\
$g_{12}$ & 1.04 & 0.407 & 0.585 & -0.157 \\
$g_{14}$ & 0.288 & 0.113 & 0.163 & -0.0405 \\
$h_{11}$ & -1.63 & -0.640 & 0.410 & 0.229 \\
$h_{12}$ & 0.157 & 0.0615 & -0.0394 & -0.0220 \\
$h_{14}$ & 1.26 & 0.495 & -0.317 & -0.177 \\
$g_{21}$ & 0.103 & 0.232 & 0.0470 & 0.0654 \\
$g_{22}$ & -0.691 & -1.56 & -0.316 & -0.438 \\
$g_{23}$ & 0.656 & 1.48 & 0.299 & 0.416 \\
$h_{21}$ & 0.375 & 0.845 & 0.171 & -0.451 \\
$h_{22}$ & -0.504 & -1.13 & -0.230 & 0.606 \\
$h_{23}$ & 0.0622 & 0.140 & 0.0284 & -0.0749 \\
$\alpha_{1}$ & 1.23 & 0.481 & 0.692 & -0.172 \\
$\beta_{1}$ & -1.23 & -0.481 & 0.308 & 0.172 \\
$\alpha_{2}$ & 0.544 & 1.23 & 0.248 & 0.345 \\
$\beta_{2}$ & -0.544 & -1.23 & -0.248 & 0.655 \\
\hline
\end{tabular}

${ }^{2}$ The logarithmic gains of $V_{-i}$ are equal to those of $V_{i}$.

${ }^{b}$ The sensitivities of $V_{-i}$ are equal to those of $V_{i}$. 
TABLE 7

Complete Characterization of the Amphibolic Pathway in Figure 2

Near Equilibrium $(\Gamma=1.11)$ Using the $S$-System Representation Generated by the Irreversible Strategy

\begin{tabular}{cccrr}
\hline & \multicolumn{4}{c}{ Dependent variables } \\
\cline { 2 - 5 } & $X_{1}$ & \multicolumn{1}{c}{$X_{2}$} & $V_{1}$ & $V_{2}$ \\
\hline Independent \\
variable & \multicolumn{4}{c}{ Logarithmic gains ${ }^{\mathrm{a}}$} \\
$X_{3}$ & 0.327 & 0.740 & -9.33 & -9.33 \\
$X_{4}$ & 0.673 & 0.260 & 9.41 & 9.41 \\
Parameter & & \multicolumn{4}{c}{ Parameter sensitivities } & \\
& & & \\
$g_{11}$ & -1.52 & -0.589 & -21.3 & -21.3 \\
$g_{14}$ & 1.55 & 0.600 & 21.7 & 21.7 \\
$h_{11}$ & -1.22 & -0.470 & 34.6 & -17.0 \\
$h_{12}$ & 1.19 & 0.460 & -33.9 & 16.6 \\
$g_{21}$ & 0.469 & 1.06 & -13.4 & 38.3 \\
$g_{22}$ & -0.458 & -1.04 & 13.1 & -37.4 \\
$h_{22}$ & -0.729 & -1.65 & 20.8 & 20.8 \\
$h_{23}$ & 0.720 & 1.63 & -20.5 & -20.5 \\
$\alpha_{1}$ & 0.0235 & 0.00911 & 0.329 & 0.329 \\
$\beta_{1}$ & -0.0235 & -0.00911 & 0.671 & -0.329 \\
$\alpha_{2}$ & 0.00907 & 0.0205 & -0.259 & 0.741 \\
$\beta_{2}$ & -0.00907 & -0.0205 & 0.259 & 0.259 \\
\hline
\end{tabular}

${ }^{\text {a }}$ The logarithmic gains of $V_{-i}$ are equal to those of $V_{i}$.

${ }^{\mathrm{b}}$ The sensitivities of $\boldsymbol{V}_{-i}$ are equal to those of $V_{i}$.

relatively low will be more robust. It will provide an accurate representation over larger variations than would be possible with high parameter sensitivities. This is confirmed by an examination of the results in Table 3. Because the parameter sensitivities are relatively low (see Table 5), the pathway can be subjected to rather large changes in operating conditions and yet the parameters of the representation remain relatively constant. Accordingly, the $\mathrm{S}$-system representation generated by the reversible strategy is able to predict accurately the bchavior of the amphibolic pathway over a wide range of conditions (Figure 5).

Complete characterization of the amphibolic pathway at an operating point near equilibrium ( $\Gamma=1.11)$ according to the alternative strategies is shown in Tables 6 and 7. Although both strategies predict the same logarithmic gains in concentration, which is a direct consequence of the definition [Eq. (8)], they appear to yield different predictions for the logarithmic gains in fluxes and for parameter sensitivities. The reason is that different $V_{i}$ and $V_{-i}$ functions, and hence different parameters, are defined by each strategy. 
Nevertheless, as we have shown, one can relate the functions and parameters in one representation to those in the other and demonstrate that the alternative representations are equivalent at a given operating point. The differences in their predictions show up as the system moves away from the nominal operating point or from one operating point to another. In the case of the S-system representation based on the reversible strategy, the results hardly change (Table 6 versus Table 5). This is another manifestation of its robust character, which was discussed above and requires no further comment.

In the case of the S-system representation based on the irreversible strategy (Table 7), the results show that the parameter sensitivities tend to be higher than those of the alternative representation (Table 6), on average 8.16

\section{TABLE 8}

Complete Characterization of the Amphibolic Pathway in Figure 2 Far from Equilibrium $(\Gamma=10)$ Using the S-System Representation Generated by the Reversible Strategy

\begin{tabular}{|c|c|c|c|c|}
\hline & \multicolumn{4}{|c|}{ Dependent variables } \\
\hline & $X_{1}$ & $X_{2}$ & $V_{1}$ & $V_{2}$ \\
\hline $\begin{array}{c}\text { Independent } \\
\text { variable }\end{array}$ & \multicolumn{4}{|c|}{ Logarithmic gains ${ }^{\mathbf{a}}$} \\
\hline$X_{3}$ & 0.224 & 0.670 & 0.0922 & 0.194 \\
\hline$X_{4}$ & 0.725 & 0.287 & 0.0112 & -0.0116 \\
\hline Parameter & \multicolumn{4}{|c|}{ Parameter sensitivities ${ }^{b}$} \\
\hline$g_{11}$ & -0.574 & -0.227 & -0.280 & 0.00921 \\
\hline$g_{12}$ & 0.215 & 0.0850 & 0.105 & -0.00345 \\
\hline$g_{14}$ & 0.405 & 0.160 & 0.198 & -0.00650 \\
\hline$h_{11}$ & -1.31 & 0.518 & 0.178 & 0.0210 \\
\hline$h_{12}$ & 0.0299 & 0.0118 & -0.00407 & -0.000481 \\
\hline$h_{14}$ & 1.26 & 0.501 & -0.172 & -0.0203 \\
\hline$g_{21}$ & 0.102 & 0.306 & 0.0420 & 0.103 \\
\hline$g_{22}$ & -0.114 & -0.341 & -0.0469 & -0.115 \\
\hline$g_{23}$ & 0.00 & 0.00 & 0.00 & 0.00 \\
\hline$h_{21}$ & 0.147 & 0.439 & 0.0604 & -0.133 \\
\hline$h_{22}$ & -0.131 & -0.392 & -0.0539 & 0.119 \\
\hline$h_{23}$ & 0.00 & 0.00 & 0.00 & 0.00 \\
\hline $\boldsymbol{\alpha}_{1}$ & 1.60 & 0.634 & 0.782 & -0.0257 \\
\hline $\boldsymbol{\beta}_{1}$ & -1.60 & -0.634 & 0.217 & 0.0257 \\
\hline$\alpha_{2}$ & 0.522 & 1.56 & 0.215 & 0.526 \\
\hline$\beta_{2}$ & -0.522 & -1.56 & -0.215 & 0.474 \\
\hline
\end{tabular}

${ }^{a}$ The logarithmic gains of $V_{-i}$ are equal to those of $V_{i}$.

${ }^{b}$ The sensitivities of $V_{-i}$ are equal to those of $V_{i}$. 
TABLE 9

Complete Characterization of the Amphibolic Pathway in Figure 2 Far from Equilibrium $(\Gamma=10)$ Using the S-System Representation Generated by the Irreversible Strategy

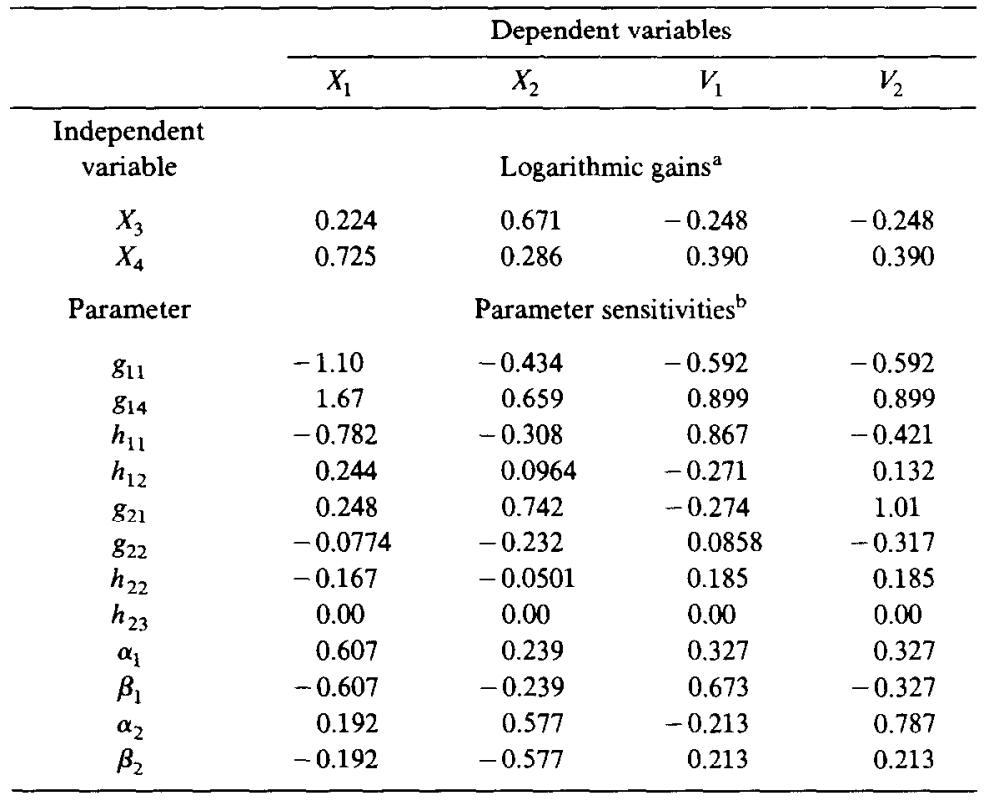

${ }^{a}$ The logarithmic gains of $V_{-i}$ are equal to those of $V_{i}$.

${ }^{\mathrm{b}}$ The sensitivities of $V_{-i}$ are equal to those of $V_{i}$.

versus 0.489 . Hence, we expect the representation bascd on the irreversible strategy to be less robust. This is confirmed by an examination of the results in Table 4. Because the parameter sensitivities are relatively high (see Table 7 vcrsus Table 6), the parameters of the representation in this case experience considerable change as the operating conditions of the pathway are altered (contrast the results in Table 4 with those in Table 3). Accordingly, the S-system representation based on the irreversible strategy is unable to accurately predict the behavior of the amphibolic pathway over as wide a range of conditions as the alternative representation (Figure 5).

Complete characterization of the amphibolic pathway at operating points further removed from equilibrium (Tables 8 and 9) shows that the differences tend to diminish (average parameter sensitivities 0.316 vs. 0.414), although the representation based on the reversible strategy always remains superior (see also Figure 5e). The reason for this convergence, as noted earlier, is that the system becomes essentially an irreversible pathway when 
the displacement from equilibrium is large, and the representation of an irreversible pathway is the same by either strategy.

\section{DISCUSSION}

Previous results $[35,36,38,39,41]$ have shown that the S-system variant within BST is superior to others such as the generalized mass action variant upon which other theories of biochemical systems are based (e.g., [4, 15]). Even within the preferred S-system representation there are alternatives that become most apparent in the analysis of amphibolic pathways. For this reason we have focused attention on this class of pathways in order to distinguish clearly the two most common strategies of representation. The "reversible" strategy involves aggregation of incoming fluxes separately from outgoing fluxes for each metabolite to define a net flux that can be positive, negative, or zero; this amounts to choosing the set of fluxes with the same sense toward a given metabolite as the building block for construction of the S-system representation. The "irreversible" strategy involves aggregation of forward and reverse fluxes through each reaction to define a net flux that is always positive; this amounts to choosing the net flux through a given reaction as a building block. There are three principal results presented in this paper.

First, we have demonstrated that all S-system representations predict the same changes in dependent concentrations for a given change in an independent concentration. Second, we have shown that the S-system representation based on the reversible strategy is superior to that based on the irreversible strategy according to several criteria, including (1) accuracy in predicting steady-state flux, (2) accuracy in predicting transient responses, and (3) robustness of the representation. Third, we have seen that only the reversible strategy yields a representation that is able to capture the principal characteristic of amphibolic pathways; namely, the reversal of net flux under physiological conditions.

Generalized mass action representations based on the irreversible strategy of aggregation (e.g., see Kacser and Porteous [15] and Crabtree and Newsholme [4], and their comparison in Sorribas and Savageau [39]) are unable to capture this essential qualitative feature of amphibolic pathways. The reasons are the same as for the S-system representation based on the irreversible strategy, although this variant is otherwise superior to the generalized mass action variant $[38,39]$. Thus, not only is the S-system variant the preferred variant within BST, but for the class of mechanisms consisting of amphibolic pathways the particular S-system representation based on the reversible strategy of aggregation is clearly superior.

Finally, the results presented in this paper document the wide range of variation over which the S-system representation can accurately predict the 
behavior of intact biochemical systems, which is in agreement with other studies $[28,38,41]$ and contrary to claims that theories based on the power-law formalism are only valid for small variations $(<10 \%)$ about a steady state $[2,15,44]$.

We thank Drs. D. H. Irvine and E. O. Voit for constructive discussion and critical examination of the manuscript, and two anonymous reviewers for suggesting improvements in the presentation. This work was supported in part by grant 135-PIS from the Presidential Initiatives Fund of The University of Michigan, U.S. Public Health Service grant GM-30054 from the National Institutes of Health, and grant EET-8712756 from the National Science Foundation.

\section{REFERENCES}

1 M. Brenner and B. N. Ames, The histidine operon and its regulation, in Metabolic Pathways, 3rd ed., Vol. 5, H. J. Vogel (Ed.), Academic, New York, 1971, pp. 349-387.

2 B. Crabtree and E. A. Newsholme, Sensitivity of a near-equilibrium reaction in a metabolic pathway to changes in substrate concentration, Eur. J. Biochem. 89:19-22 (1978).

3 B. Crabtree and E. A. Newsholme, A quantitative approach to metabolic control, Current Topics Cell. Reg. 25:21-76 (1985).

4 B. Crabtree and E. A. Newsholme, The derivation and interpretation of control coefficients, Biochem. J. 247:113-120 (1987).

5 B. D. Davis, The teleonomic significance of biosynthetic control mechanisms, Cold Sp. Harbor Symp. Quant. Biol. 26:1-10 (1961).

6 D. A. Fell and H. M. Sauro, Metabolic control and its analysis, Eur. J. Biochem. 148:555-561 (1985).

7 R. Heinrich and T. Rapoport, A linear steady-state treatment of enzymatic chains, Eur. J. Biochem. 42:89-95 (1974).

8 R. Heinrich and T. A. Rapoport. Mathematical analysis of multienzyme systems 2. Steady-state and transient control, Biosystems 7:130-136 (1975).

9 D. H. Irvine and M. A. Savageau, Network regulation of the immune response: alternative control points for suppressor modulation of effector lymphocytes, $J$. Immunol. 134:2100-2116 (1985).

10 D. H. Irvine and M. A. Savageau, Network regulation of the immune response: modulation of suppressor lymphocytes by alternative signals including contrasuppression, J. Immunol. 134:2117-2130 (1985).

11 D. H. Irvine and M. A. Savageau, Efficient solution of nonlinear ordinary differential equations expressed in S-system canonical form, SIAM Journal on Numerical Analysis (in press).

12 H. Kacser, The control of enzyme systems in vivo: elasticity analysis of the steady state, Biochem. Soc. Trans. 11:35-40 (1983).

13 H. Kacser and J. A. Burns, The control of flux, Symp. Soc. Exp. Biol. 27:65-104 (1973).

14 H. Kacser and J. A. Burns, Molecular democracy: who shares the controls?, Trans. Biochem. Soc. 7:1149-1160 (1979). 
15 H. Kacser and J. W. Porteous, Control of metabolism: what do we have to measure?, Trends Biochem. Sci. 12:5-14 (1987).

16 B. Magasanik and H. R. Bowser, The degradation of histidine by Aerobacter aerogenes, J. Biol. Chem. 213:571-580 (1955).

17 H. K. Meiss, W. J. Brill, and B. Magasanik, Genetic control of histidine degradation in Salmonella typhimurium, strain LT-2, J. Biol. Chem. 244:5382-5391 (1969).

18 M. Okamoto and M. A. Savageau, Integrated function of a kinetic proofreading mechanism: steady-state analysis testing internal consistency of data obtained in vivo and in vitro and predicting parameter values, Biochemistry 23:1701-1709 (1984).

19 B. D. Sanwal, Allosteric controls of amphibolic pathways in bacteria, Bacteriol. Rev. 34:20-39 (1970).

20 M. A. Savageau, Biochemical systems analysis I. Some mathematical properties of the rate law for the component enzymatic reactions, J. Theoret. Biol. 25:365-369 (1969).

21 M. A. Savageau, Biochemical systems analysis II. The steady state solutions for an n-pool system using a power-law approximation, J. Theoret. Biol. 25:370-379 (1969).

22 M. A. Savageau, Biochemical systems analysis III. Dynamic solutions using a powerlaw approximation, $J$. Theoret. Biol. 26:215-226 (1970).

23 M. A. Savageau, Parameter sensitivity as a criterion for evaluating and comparing the performance of biochemical systems, Nature 229:542-544 (1971).

24 M. A. Savageau, Concepts relating the behavior of biochemical systems to their underlying molecular properties, Arch. Biochem. Biophys. 145:612-621 (1971).

25 M. A. Savageau, The behavior of intact biochemical control systems, Current Topics Cell. Reg. 6:63-130 (1972).

26 M. A. Savageau, Optimal design of feedback control by inhibition: steady-state considerations, J. Mol. Evolution 4:139-156 (1974).

27 M. A. Savageau, Optimal design of feedback control by inhibition: dynamic considerations, J. Mol. Evolution 5:199-222 (1975).

28 M. A. Savageau, Biochemical Systems Analysis: A Study of Function and Design in Molecular Biology, Addison-Wesley, Reading, Mass., 1976.

29 M. A. Savageau, Growth of complex systems can be related to the properties of their underlying determinants, Proc. Nat. Acad. Sci. USA 76:5413-5417 (1979).

30 M. A. Savageau, Autogenous and classical regulation of gene expression: a general theory and experimental evidence, in Biological Regulation and Development, Vol. 1, R. F. Goldberger (Ed.), Plenum, New York, 1979, pp. 57-108.

31 M. A. Savageau, Coupled circuits of gene regulation, in Sequence Specificity in Transcription and Translation, R. Calendar and L. Gold (Eds.), Alan R. Liss, New York, 1985, pp. 633642.

32 M. A. Savageau, Control of metabolism: where is the theory?, Trends Biochem. Sci. 12:219-220 (1987).

33 M. A. Savageau and G. Jacknow, Feedforward inhibition in biosynthetic pathways: inhibition of the aminoacyl-tRNA synthetase by intermediates of the pathway, J. Theoret. Biol. 77:405-425 (1979).

34 M. A. Savageau and A. Sorribas, Constraints among molecular and systemic properties: implications for physiological genetics (submitted).

35 M. A. Savageau, E. O. Voit, and D. H. Irvine, Biochemical systems theory and metabolic control theory 1. Fundamental similarities and differences, Math. Biosci. 86:127-145 (1987).

36 M. A. Savageau, E. O. Voit, and D. H. Irvine, Biochemical systems theory and metabolic control theory 2 . The role of summation and connectivity relationships, Math. Biosci. 86:147-169 (1987). 
37 A. Sorribas, Discovering another view of control analysis, Trends Biochem. Sci. 12:221-222 (1987).

38 A. Sorribas and M. A. Savagcau, A comparison of variant theorics of intact biochcmical systems I. Enzyme-enzyme interactions and biochemical systems theory, Math. Biosci., 94:161-193 (1989).

39 A. Sorribas and M. A. Savageau, A comparison of variant theories of intact biochemical systems II. Flux oriented and metabolic control theories, Math. Biosci., 94:195-238 (1989).

40 E. O. Voit, Control in perspective, Trends Biochem. Sci. 12:221 (1987).

41 E. O. Voit and M. A. Savageau, Accuracy of alternative representations for integrated biochemical systems, Biochemistry 26:6869-6880 (1987).

42 G. R. Welch and T. Keleti, Is cell metabolism controlled by a "molecular democracy" or by a "supramolecular socialism"?, Trends Biochem. Sci. 12:216-217 (1987).

43 H. V. Westerhoff and Y.-D. Chen, How do enzyme activities control metabolic concentrations?, Eur. J. Biochem. 142:425-430 (1984).

44 H. V. Westerhoff and D. B. Kell, Matrix method for determining steps most rate limiting to metabolic fluxes in biotechnological processes, Biotechnol. Bioeng. 30:101-107 (1987). 